\title{
Developing Model for Supply Chain Management - the Case of Croatia
}

E. Jurun, I. Veza

This paper describes a model of supply chain management (SCM). It explains overall supply chain issues, strategic importance of SCM, supply chain strategies and an example of mathematical formulation. A supply chain is a global network of organizations that cooperate to improve the flows of material and information between suppliers and customers at the lowest cost and the highest speed. The objective of a supply chain is customer satisfaction. At the strategic level, a supply chain can be considered as being composed of five activities: buy, make, move, store and sell. Each activity is a module. The set of modules, along with its links, constitutes a model of the supply chain. Our paper presents some insights into the supply chain strategies of companies in Croatia. The major goal of this paper is to show a model for supply chain management in mathematical terms, with an example of mathematical formulation.

Keywords: supply chain, supply chain management, system modeling, logistic system, supply chain strategies.

\section{Introduction}

At the turn of the third millennium, world industry finds itself in probably the largest restructuring since the first industrial revolution. The progress is determined by two trends:

- dynamic progress of information and communication technologies, which have enabled the creation of new markets and a redefinition of entire professions,

- globalization of the economy thanks to new purchasing and selling markets.

Such progress forces enterprises to modify their production strategies. New competitors and greater changes in demand in the course of time in a stagnant market are presenting enormous cost pressure for many enterprises. In order to meet customers' needs everywhere in the world, the enterprises should increase their flexibility.

The main requirements put upon industry are the following:

1. Cost pressure:

- stagnant markets,

- great changes in demand,

- producers with a cheap work force.

2. economic globalization:

- new markets for sales (Asia/Eastern Europe),

- new purchasing markets,

- new competitors.

3. The third industrial revolution:

- new potentials obtained by the application of information and communication technologies,

- structural changes in entire professions,

- new products and markets.

Nowadays, the main conditions for a successful enterprise are as follows:

- the existence of products/ideas/services,

- the existence of a market,

- maximum fulfilment of customers' requirements,

- minimum use of resources.
Long-lasting success will be achieved only by enterprises which not only achieve the necessary optimization of their production process but also identify and conquer new markets.

One possible conception for survival in a turbulent world market is chain cooperation involving the producer, his suppliers and customers, according to the concept of Supply Chain Management.

\section{The basics of supply chain management}

Supply Chain Management (SCM) comprises process-oriented integration of planning, proceeding, coordination and control of material and information flows in a one-stage or multi-stage supply chain in the area of planning, purchasing, production and distribution.

The basic idea of SCM is a further development of supply logic. Classical supply management is engaged in optimizing material and information flows in a particular enterprise, while SCM focuses on the entire integration of all partners in a supply chain. The aim of SCM is to reduce costs in the entire production process, from raw materials purchasing to the delivery to the final customer, optimizing all partners in a chain. The partners in a supply chain can be departments of either one enterprise or single enterprise.

A number of SCM initiatives have already been taken, SCOR being the most important of them. This represents a new generation of SCM systems. Supply Chain Operation Reference (SCOR) was founded in the USA in 1996, by the Supply Chain Council (SCC), as an independent organization (Web pages http://www.supply-chain.org). It uses SCM systems and further improves them. The SCOR model is a significant auxiliary device, which standardizes cooperation processes among several enterprises and makes them transparent. The main aim of the SCOR initiative is business process modelling, which takes place among several enterprises in a Supply Chain, so that SCM can be realized. The processes are comparable by the process reference model. The SCOR model consists of four fundamental processes: PLAN, SOURCE, MAKE and DELIVER, which are inter- 


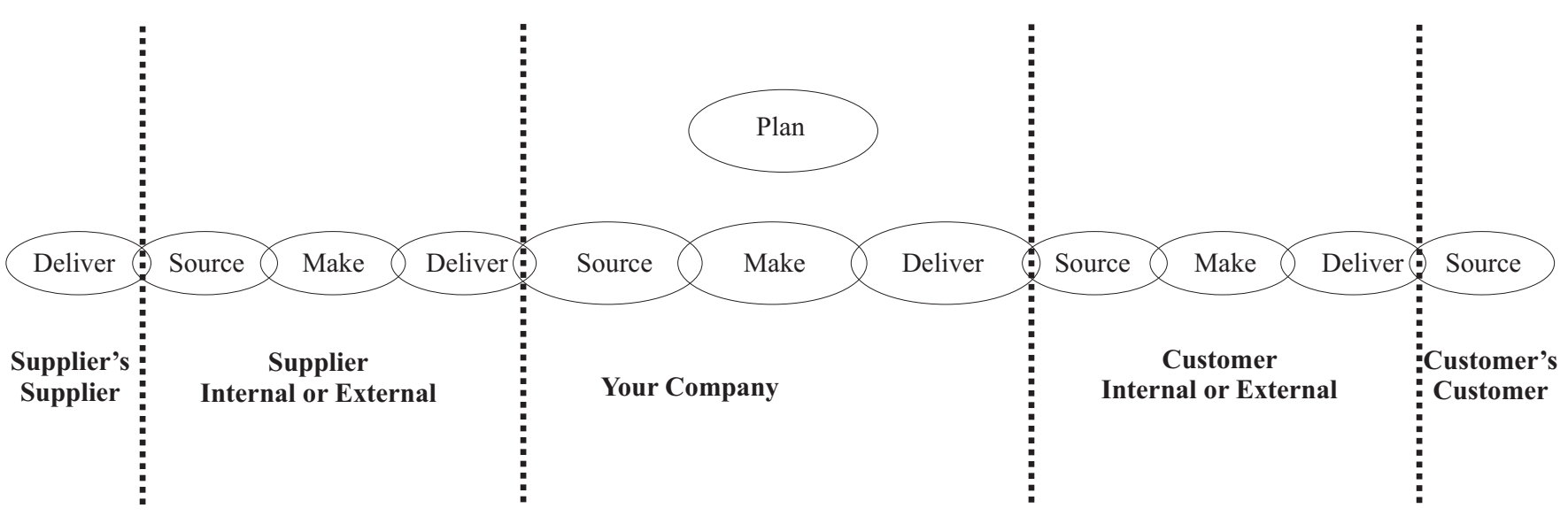

Fig. 1: The basis of the SCOR reference model (Top-Level) [1]

linked in a chain (Fig. 1.). The figure shows the interconnection in the chain between supplier, producer and distributor in order to achieve the common optimum with reference to costs, delivery time, delivery amount and inventories.

According to the definition, SCM contains the following potentials:

- supplier service improvement,

- reduction of net inventories,

- shortening of the entire production cycle,

- enhancement of predicting accuracy,

- a rice in productivity,

- lower costs for purchasing, production and distribution in the chain.

By applying the SCM concept it is possible to reduce inventories up to $60 \%$, shorten the production cycle up to $50 \%$, increase profit up to $30 \%$ and total costs up to $25 \%$. Thus, for example, the Coca Cola Company, after introducing SCM and using potentials for rationalization, has achieved a 3,5\% increase in profit in the European market $[2,3]$.
With regard to the planned horizon and planning objects, global and local planned assignments within SCM are divided into three time and logic levels:

- Strategy level. The main task of the strategic level of planning is to define the enterprise strategy by shaping a configurationally optimal production and supply network among several enterprises. On the basis of alternative configurations, a simulation with reference to given criteria chooses an optimal solution. Distribution channels, from the raw material supplier to the selling market, are analysed in this phase. This is done on the basis of the annual planned quantity, production quantity and the situation on the stock. The aim of shaping is to get a deliverer's real supply chain with reference to all relevant limits.

- Tactical level. Based on the data obtained on the strategic level, particular members of the production network, with reference to long-term production and transport plans, are defined in this phase. The aim of planning is to synchronize a medium-term and long-term planning programme with reference to capacities and terms (between 3 and 6

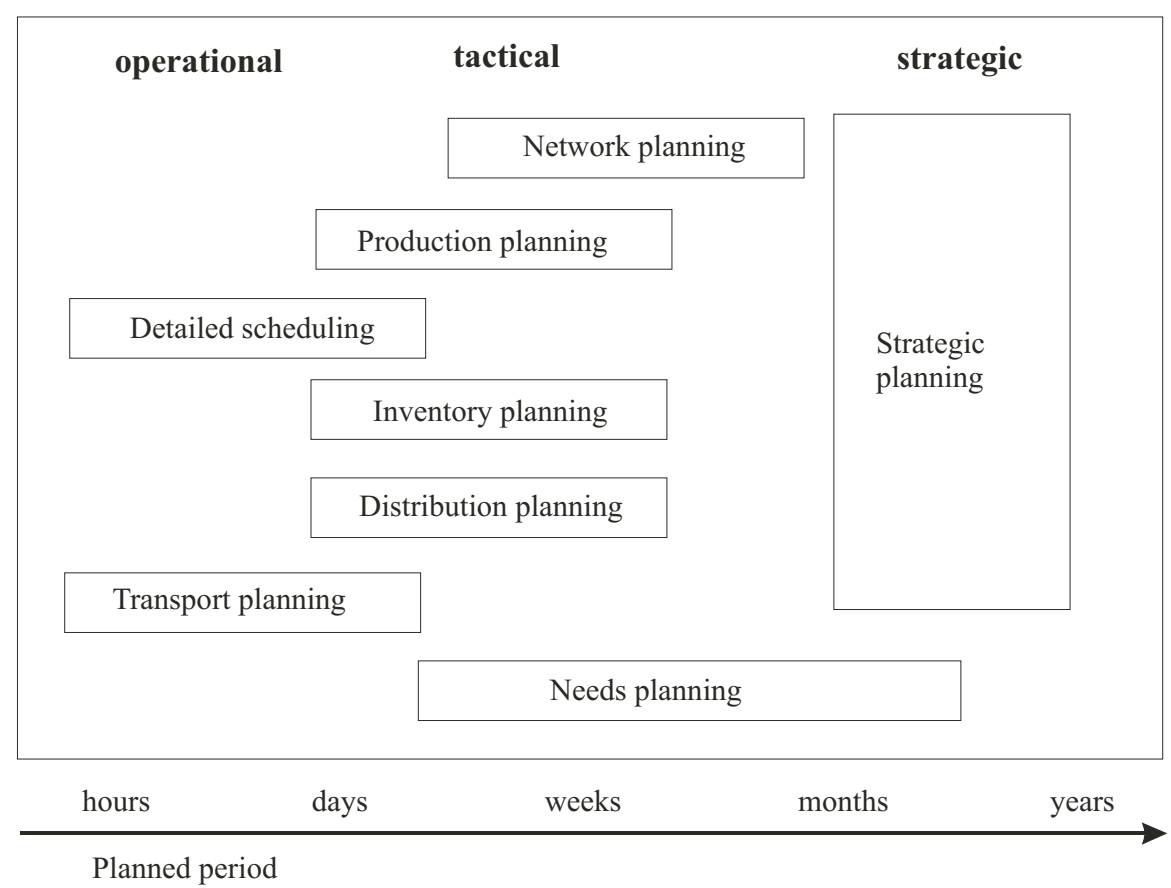

Fig. 2: Planned assignments with regard to the planned period 
months). The input data for this planning are necessary information on the Supply Chain structure, selling predictions and customers' needs. Rough planning of purchasing, production and distribution is done on the basis of the input data. Planning is done by means of simulation of different alternatives with reference to resource, costs and delivery times.

- Operational level. Operational realization of the set programmes takes place through production planning and control (PPC). The existing PPC organizational structures can be used to realize SCM, but they have to be extended in dependence on the exterior partner. It is indispensable to ensure quick exchange of information between the supplier and the customer so that a quick reaction to unplanned events (e.g. disturbances, short-term special orders and others) can be provided. Typical planned functions at the operational level are detailed scheduling (on the basis of plans at the tactical level) and controlling of orders, warehouse and transport.

Planned assignments with regard to the planned period are presented in Fig. 2.

The major goal of this paper is to show a model for supply chain management in mathematical terms with an example of mathematical formulation.

\section{Model for supply chain management}

In most industries the cost of raw materials and component parts from external vendors constitutes the main part of the total product cost, and in some cases it can account for up to $80 \%$ of the total product cost. For this reason, supplier selection is one of the most important tasks in every industry.

Basically there are two kinds of supplier problems; the first is supplier selection when there is no constraint. In other words when all suppliers can satisfy the buyer's requirements for demand, quality, delivery, etc. In this kind of supplier selection the management needs to make only one decision which supplier is the best.

The second type of supplier selection problem is when there are some limitations on suppliers capacity, quality and so on. In other words, no supplier can satisfy the buyer's total requirements and the buyer needs to purchase partly from one supplier and partly from another to compensate for the shortage of capacity or low quality of the first supplier. The enterprise must decide which supplier it should contract, and it must determine the appropriate order quantity for each supplier selected.

This paper will present a cost minimizing model for supplier selection with price breaks. The existence of variable prices offered by the supplier usually complicates the selection process for the purchaser [5]. The change in prices depends upon the size of the order. In cases when variable prices are combined with capacities or limited conditions of delivery, supplier selection can be very complicated.

Consider a buyer who requires an amount $D$ of a particular product over a fixed planning period. There are $n$ suppliers that can meet the buyer's demand. Let $x_{i}$ be the order quantity of this product placed with supplier $i$, where

$$
\sum_{i=1}^{n} x_{i}=D
$$

Supplier $i$ can provide up to $v_{i}$ units of the product over the planning period, i.e.

$$
x_{i} \leq v_{i}
$$

It is assumed that the aggregate amount of the product available from this multiple-sourcing network is sufficient to satisfy $D$, i.e.

$$
\sum_{i=1}^{n} v_{i} \geq D
$$

Depending on the type of product, aggregate supplier performance measures of quality and delivery may or may not be meaningful to the buyer. We assume that they are in this section, for the purpose of illustration. The order quantity must therefore satisfy:

$$
\sum_{i=1}^{n} q_{i} x_{i} \geq Q D \text { and } \sum_{i=1}^{n} l_{i} x_{i} \leq L D
$$

where:

$q_{i} \quad$ quality of a unit of the product of supplier $i$,

$Q \quad$ buyer's minimum quality requirement for a unit of a product,

$l_{i} \quad$ lead time from the supplier that is needed to fulfill an order,

$L \quad$ deadline, measured from the order date.

Let $p_{i}\left(x_{i}\right)$ be the price that the buyer must pay to supplier $i$ for supplying the order quantity $x_{i}$. Assume that the suppliers offer some type of price breaks. The net price that the buyer must pay for $D$ is then $\sum_{i=1}^{n} p_{i}\left(x_{i}\right)$, which the buyer desires to minimize.

The supplier selection problem of the buyer may then be modeled by the following mathematical program:

$\min \sum_{i=1}^{n} p_{i}\left(x_{i}\right)$ (objective: minimize the buyer's price for ordering $D$ )

$\sum_{i=1}^{n} q_{i} x_{i} \geq Q D$ (aggregate quality constraint)

$\sum_{i=1}^{n} l_{i} x_{i} \leq L D($ aggregate delivery constraint $)$

$\sum_{i=1}^{n} x_{i}=D($ demand constraint $)$

$x_{i} \leq v_{i} \quad i=1, \ldots, n$ (supplier constraints)

$x_{i} \geq 0 \quad i=1, \ldots, n$ (non-negativity constraint)

The objective function is nonlinear due to the presence of price breaks. A special case for supplier selection is cumulative price breaks. Suppose each supplier offers all-unit price breaks. This implies that the cost of purchasing $x_{i}$ units from supplier $i, p_{i}\left(x_{i}\right)$ is defined to be 


$$
p_{i}\left(x_{i}\right)=p_{i j} x_{i} \text { if } b_{i, j-1} \leq x_{i} \leq b_{i j}, 1 \leq j \leq m(i)
$$

where:

$p_{i j} \quad$ is the unit price for level $j$,

$b_{i j} \quad$ are the quantities at which price breaks occur,

$m(i)$ is the number of quantity ranges in vendor is price schedule. If $p_{i j}$ is a decreasing (increasing) function of $j$, then quantity discounts are being offered.

We define a more specific set of decision variables that describes the order quantities:

$x_{i j} \quad$ is the number of units purchased from supplier $i$ at price level $j$.

It follows that $x_{i}$ is related to these $x_{i j}$ by

$$
x_{i}=\sum_{j=1}^{m(i)} x_{i j}, \quad i=1, \ldots, n .
$$

Since $x_{i j}$ can be nonzero only if

$$
b_{i, j-1} \leq x_{i j} \leq b_{i j}
$$

it follows that the objective function becomes

$$
p_{i}\left(x_{i}\right)=p_{i j} x_{i j}
$$

which is nonlinear.

To circumvent this nonlinearity, we introduce binary integer variables $y_{i j}$ in the following way:

$$
y_{i j}=\left\{\begin{array}{lll}
0 & \text { if } & x_{i j}=0 \\
1 & \text { if } & x_{i j}>0
\end{array} .\right.
$$

This definition can be realized by adding constraints of the form

$b_{i, j-1} y_{i j} \leq x_{i j} \leq b_{i j}^{*}, j=1, \ldots, m(i)$ (lower and upper quantity range constraints).

$b_{i j}^{*}$ is a number slightly lower than $b_{i j}$ introduced to realize

$$
x_{i j}<b_{i j} y_{i j}
$$

for all $i$ and $j=1, \ldots, m(i)-1$, and

$$
b_{i, m(i)}^{*}=D
$$

for all $i$, since $D$ is the most any supplier can supply.

Actually, for $i=1, \ldots, n$, the constraints

$$
b_{i 0} y_{i 1} \leq x_{i 1}
$$

are redundant, since $b_{i 0}$ and $x_{i 1}$ is defined to be non-negative. A further constraint for each supplier is that at most one price level can be chosen, i.e.

$\sum_{j=1}^{m(i)} y_{i j} \leq 1, \quad i=1, \ldots, n$ (price level constraints).

The situation can be even more complex. Namely, let xijk be the number of units of the good $\mathrm{k}$ (the kind of material required) offered by supplier $\mathrm{i}$ at price level $\mathrm{j}$. At the same time there are $\mathrm{n}$ different suppliers, $(i=1, \ldots, n), l$ kinds of goods $(k=1, \ldots, l)$, and each supplier offers $m(i)(j=1, \ldots, m(i))$ price levels, where the numbers of different discount categories depends on each supplier.

Let us assume that the producer requires the total quantity of goods $D$, therefore

$$
\sum_{i=1}^{n} \sum_{j=1}^{m(i)} \sum_{k=1}^{l} x_{i j k}=D
$$

must be valid.

At the same time there are individual requirements for each kind of goods $D_{k}$, i.e. the following relations must be valid:

$$
\sum_{i=1}^{n} \sum_{j=1}^{m(i)} x_{i j k}=D_{k} \text { and } \sum_{k=1}^{l} D_{k}=D .
$$

If we consider in this way all kinds of goods required together, the model becomes extremely complex, with a great number of variables and constraints. Such a model can be subdivided into a number of small models, each to be separately solved, which makes the problem simpler.

\section{Supply chain management - the case of Croatia}

Construction of a modern and efficient economic structure is, certainly, one of the fundamental objectives for the reconstruction and development of the Republic of Croatia. It can be safely assumed that many different factors and processes, especially privatisation and restructuring, influence the realisation of such an objective.

Namely, privatization in Croatia is entering a new stage, which is characterized by the further privatization of small and medium-sized enterprises, as well as the end of privatization of large production systems, banks and public enterprises. Restructuring is oriented toward the creation of a network of small and medium-sized enterprises, characterized by a high degree of flexibility, adaptability and creativity. They should be based on the principles of entrepreneurial economics and entrepreneurial spirit. Restructuring is being carried out at all levels, especially at the level of large and complex systems.

The Republic of Croatia is a country in transition from the socialist, state-dictated economy toward market principles. Such a process implies a need for overall transformation of Croatian companies, which includes all segments of the organizational restructuring process.

All these factors are also related to the need for the internationalization of Croatian enterprises, and by the imperative to be included in the international processes of economic cooperation and globalization.

Croatian companies are facing severe competition, on both the international, and the domestic market, which is becoming more and more dynamic and complex. In such a context, Supply Chain Management (i.e. logistic chain management) becomes a significant factor that contributes to the creation of competitive advantage, which can be justified by the costs of logistics in the total costs of goods produced in Croatia (which is estimated to be higher than $40 \%$ ).

Threats from the environment, as well as rising competition, require Croatian enterprises to utilize new strategies of logistics and distribution, with the objective to optimize the flows of both goods and materials - from their source to the final consumer. Such an objective is to be implemented with the idea of partnership in all the stages of the supply chain.

The supply chain philosophy is based on partnership among all those involved. Instead of the traditional competition, the supply chain is being built upon the concept of 
"co-operation", which aims to mutually maximize the profit of all members of the supply chain. Lowering the costs and improving the quality of the shared logistics - e.g., by better planning of the materials and goods requirements, by utilization of contemporary information technology in information exchange, etc., - can achieve this.

Even in Croatian enterprises, the traditional approach to competitive advantage usually referred to as the 4Ps (product, price, promotion and place - i.e. the philosophy of a quality product as an acceptable price, with appropriate promotion and found in the right place) is being replaced by the $3 \mathrm{R}$ concept (referring to reliability, responsiveness and relationships).

This concept is based on the principles of reliable and responsive delivery, as well as on stable relationships. Such a concept is being increasingly used by a range of Croatian enterprises. AD Plastik d.d. can be identified as one such enterprise, as evidenced by its long-term cooperation with Renault and Peugeot.

\section{Conclusion}

With regard to the development trend of supplier chains between enterprises in different locations, today in Europe, and particularly in the USA, inter-enterprises networking is constantly increasing. In the USA there are already well known examples of networking among several enterprises (e.g. Wall Mart trade work chain).These potentials are available for those who introduce SCM should be used in Croatian enterprises in the future. In order to realise this, it is necessary to network enterprises in a particular region and to connect them, for the purpose of knowledge transfer, with science and research institutions. An important role in this network should be played by state institutions (economic chambers, regional and municipal government, etc.) which should decide on a development strategy and projects for faster transfer to a market economy and entry into European integration.

\section{References}

[1] Alard R., Hartel I., Hieber, R.: "Innovationstreiber im Supply Chain Management”,, io Management Vol. 5 (1999), p. 64-67.

[2] Beckmann H.: "Integrale Logistik als Wachstumskonzept - Supply Chain Management - Strategie der Kooperation". In: Jahrbuch Logistik 1998 (Editors: K. Radeker and V. Kirsten). Düsseldorf: VDI, 1998, p. 23-29.

[3] Dinges M.: "Supply Chain Management - Logistikrevolution oder Wein in alten Schläuchen?" Information Management \& Consulting , Vol. 13 (1998), No. 3, p. 22-27.

[4] Kansky D.: "Supply Chain Management". Industrie Management, Vol. 15,(1999), No. 5, p. 14-17.

[5] Jurun E., Babic Z., Tomic-Plazibat N.: "A Model Approach to the Vendor Selection Problem". Proceedings of the $8^{\text {th }}$ Conference on Operational Research KOI 2000, Mathematical Communications, Vol. 1, (2001), No.1, p.103-110.

Elza Jurun, Ph.D.

Želimir Dulcic, Ph.D.

University of Split

Faculty of Economics

Matice hrvatske 31

21000 Split, Croatia

Ivica Veza, Ph.D.

University of Split

Faculty of Electrical Engineering,

Mechanical Engineering and Naval Architecture

R. Boskovica bb

21000 Split, Croatia 